\title{
A practical heparin reduction algorithm: execution and operational characteristics
}

\author{
HERMAN E BRANSON, DAVED VAN STRALEN, JOANNE SCHOTTINGER, \\ WALTER PETERS, YASUYUKI ENDO* \\ From the Departments of Pathology and Internal Medicine, University of California, Irvine, California
}

SUMMARY A limited pilot study has been made of a newly devised heparin reduction algorithm (HRA). This formulation is a derivative of the alternative surveillance plan known as the activated partial thromboplastin time after heparin removal (aPTT/HR) scheme. Unlike the traditional plan, the HRA is the first approach to provide information about the individual and collective pharmacological effects of heparin and coumarins when the drugs are administered simultaneously. In this feasibility study the HRA was used without incident in six patients every $24 \mathrm{~h}$ to calculate the trend of the evolving anticoagulant effect of coumarin. The computations provided by a laboratory based data management group permitted the clinician to titrate precisely the withdrawal of heparin in response to the daily fluctuations in coumarin effect. In this way, the activated partial thromboplastin time could always be maintained within the desired therapeutic interval. Three divergent patient experiences are presented to demonstrate the operational characteristics and responsiveness of the new HRA plan.

Before the development of anion exchange procedures, the critical assessment of the anticoagulant effect of coumarins in the presence of heparin was dependent on the expensive and biasing techniques of neutralisation and dilution..$^{-3}$ In 1979 Branson et al showed that the presence of coumarin modified dyszymogens in no way compromised the removal of heparin from plasma samples by ECTEOLA (epichlorohydrin triethanolamine) cellulose columns ${ }^{4}$ in experiments employing the one stage prothrombin time (PT). The new test known as the prothrombin time after heparin removal (PT/HR) was found to be at least as effective as dilutional prothrombin times of the Owren type for following the transition from heparin to coumarins. The PT/HR has the additional advantages of being: (a) applicable to all prothrombin time reagent systems; $(b)$ sensitive to low levels of fibrinogen and/or factor V; and (c) less expensive.

While the anion exchange prothrombin time

Accepted for publication 3 January 1985

*Present address: The Third Department of Internal Medicine, Akita University School of Medicine, 1-1-1 Hondo Akita 010, Japan.
(AEPT) is a superior replacement for dilutional prothrombin times, application of heparin removal technology to the activated partial thromboplastin time (aPTT) is a far more powerful combination..$^{5}$ The $\mathrm{aPTT} / \mathrm{HR}$ is the cornerstone of an alternative anticoagulant surveillance plan-that is, the aPTT/ HR scheme. In the new approach the antithrombotic actions of heparin and coumarins are equated to seconds prolongation of the same test-that is, aPTT-whether the drugs are administered alone or in combination. Several aPTT reagents suitable for the application have been identified.

These factors have contributed to the formulation of a heparin reduction algorithm (HRA) based on the aPTT/HR. The HRA is designed to permit the physician managing a victim of venous thromboembolic disease to withdraw heparin in rational decrements in direct proportion to the amount of anticoagulant protection generated by the administration of vitamin $\mathrm{K}$ antagonists. The new approach is designed to replace the empirical discontinuance of heparin, now used universally, during the most treacherous interval of anticoagulant treatment. ${ }^{6}$ Recently, a limited pilot trial was conducted of the HRA to establish the operational characteristics and responsiveness of the plan in different cases. 


\section{Patients and methods}

\section{STUDY DESIGN}

A prospective pilot study was conducted to determine the feasibility of adjusting heparin dosage in response to the coumarin anticoagulant effect by means of the HRA. The project was approved by the Human Subject Review Committee of the University of California, Irvine.

Six patients, aged 24 to 68 , who were considered eligible by participating physicians to receive three phase antithrombotic treatment, were admitted to the study over a three month period. Patients who had been over their acute illness for at least three days and who were ready to start oral treatment within $48 \mathrm{~h}$ were included in the investigation. Heparin was given to all patients by the constant infusion method. All decisions about heparin treatment - that is, dosage, infusion rate, etc-were made by the clinician. An interval of optimal heparin effect was defined for the purposes of making calculations with the HRA which served as a target level for coumarin antithrombotic protection. Generally, the amount of heparin protection afforded by the dosage $24 \mathrm{~h}$ before the first dose of coumarin was considered optimal.

During the transition interval each physician managing a study group patient was provided with the results of the aPTT, aPTT/HR, PT, and PT/HR on at least a daily basis or more often, on request. A suggested percentage heparin reduction was calculated for each patient by a data management team of physicians, students, and coagulation technologists at the beginning of the day. Thus the clinician could oversee the adjustment of the heparin infusion apparatus. The amount of heparin ordered and the amount actually infused were carefully monitored. No attempt was made to control the amount of coumarin administered to study group patients. The HRA formula employed during the study was:

$$
\% \text { heparin reduction }=\frac{\mathrm{aPTT} / \mathrm{HR}^{\mathrm{n}}-\mathrm{aPTT} / \mathrm{HR}^{\mathrm{n}-1}}{\mathrm{aPTT^{0 }}-\mathrm{aPTT} / \mathrm{HR}^{\mathrm{o}}} \times 100
$$

where $n-1=$ preceding interval and $o=$ interval of optimal heparin effect.

Therapeutic ranges for coumarins were calculated to be between 1.3 and 2.5 times the $\mathrm{aPTT} / \mathrm{HR}_{\mathrm{o}}$ of each patient. After the transition from heparin to

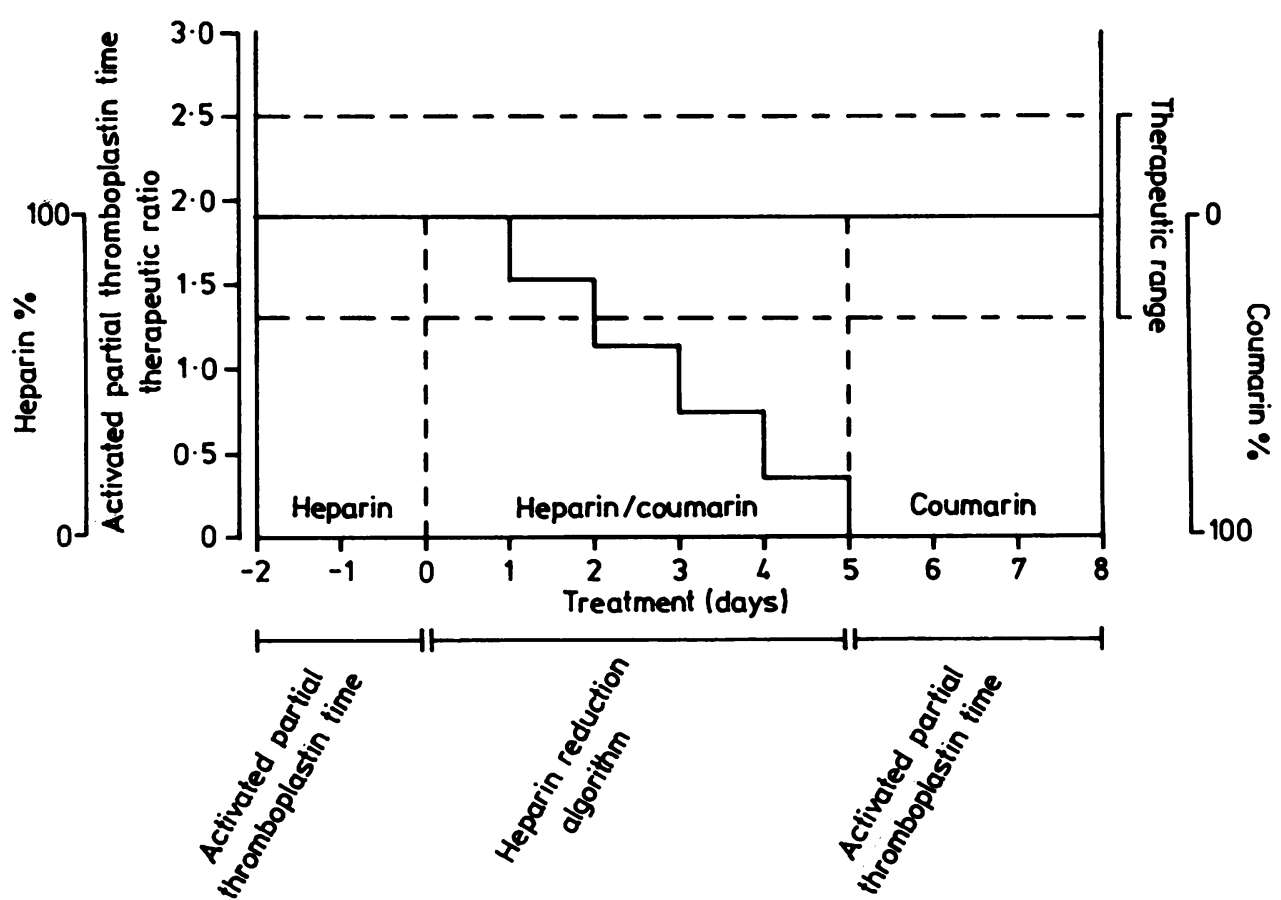

The ideal execution of the heparin reduction algorithm is illustrated in a hypothetical patient being maintained in the middle of the therapeutic range at 1.9 times the baseline activated partial thromboplastin time. Oral anticoagulant treatment is started on day 0 . A cycle time of 24 h is shown with a uniform increase in the coumarin percent total anticoagulant protection of $20 \% / 24 \mathrm{~h}$ (right ordinate). The heparin dosage increment is decreased in reciprocal fashion (left ordinate) each day until discontinued on day 5. 
coumarin protection was effected, the protocol was completed and the patients were discharged to be followed by means of the prothrombin time. The experiences of three of the six patients managed by means of the HRA are presented.

\section{CASE REPORTS}

\section{Patient 3}

A 67 year old obese $(101 \mathrm{~kg})$ woman with breast cancer metastatic to the lungs and bilateral chronic venous stasis of the lower extremities was admitted to the hospital after presenting with acute swelling and tenderness of the right calf and thigh of about $36 \mathrm{~h}$ duration. Venography confirmed deep venous thrombophlebitis, which was treated with continuous infusion heparin. The patient had been admitted to hospital on three separate occasions for thrombophlebitis without demonstrable pulmonary embolism. The platelet count, PT, and aPTT were normal on this admission. Her acute symptoms resolved within $12 \mathrm{~h}$. She was discharged on the 17 th day on warfarin sodium $5 \mathrm{mg} / 24 \mathrm{~h}$.

\section{Patient 4}

A 68 year old $(72 \mathrm{~kg})$ man with metastatic colon carcinoma and intractable pain was admitted for elective neurosurgical palliation procedures. On the 34th day in hospital, for the first time, the patient developed swelling of the left leg with positive phlebogram findings. The platelet count was $440 \times$ $10^{y} / 1$ while all other laboratory studies were within normal limits. He responded rapidly to heparin and was placed on coumarin after 10 days. The transition to warfarin sodium took eight days.

\section{Patient 5}

A 40 year old $(74.3 \mathrm{~kg})$ man with a history of one episode of post-traumatic deep venous thrombosis with pulmonary embolism was admitted to hospital for pain and swelling of the left ankle resulting from an injury four days earlier. Laboratory tests for haemostasis and liver and kidney function were normal on admission. The patient responded to heparin within $24 \mathrm{~h}$ and made the change to coumarin protection in six days.

\section{COAGULATION STUDIES}

Patient samples were collected in Monoject tubes (Sherwood Medical Division, Brunswick Co, St Louis, MO) containing 1 volume of $3.8 \%$ sodium citrate for anticoagulating 9 volumes of blood delivered by negative pressure. Samples were centrifuged at $4^{\circ} \mathrm{C}$ for $10 \mathrm{~min}$ at $4000 \mathrm{~g}$. The PT, aPTT, and assays of factors II, VII, IX, and X were performed as previously described using a photoelectronic endpoint apparatus. ${ }^{7}$ Global tests-that is, PT, aPTT, PT/HR, aPTT/HR-were performed preferentially in the study over factor assays at every sampling interval.

Laboratory mean values for the PT and aPTT were $11.2 \pm 1 \cdot 1$ and $33.1 \pm 3.3 \mathrm{~s}$, respectively. General Diagnostics (Morris Plains, NJ) APTT Reagent and Simplastin were used as described previously. ${ }^{5}$ The normal ranges for prothrombin group factors at the time of study were $100 \pm 20 \%$. The ideal therapeutic range for the aPTT (aPTT/HR) was 43.0 to $82.8 \mathrm{~s}$ and for the PT (PT/HR) 16.8 to $28.0 \mathrm{~s}$.

The ideal therapeutic range for the commercial aPTT reagent used in the study was derived by comparison with PT values and vitamin $\mathrm{K}$ factor concentrations performed on plasma from 60 patients who had received coumarin treatment. The optimal therapeutic ranges for individual study group patients were calculated from their aPTT/HRo values.

As described earlier, ECTEOLA obtained from Sigma Chemical Company (St Louis, MO) was used exclusively to remove heparin. ${ }^{8}$ The cellulosic anion

Table 1 Uncomplicated execution of the heparin reduction algorithm: patient 3

\begin{tabular}{|c|c|c|c|c|c|c|c|c|c|}
\hline Day & $\underset{(s)}{a P T T}$ & $\underset{(s)}{a P T T / H R}$ & $\begin{array}{l}h r \\
(\%)\end{array}$ & $\begin{array}{l}P T \\
(s)\end{array}$ & $\begin{array}{l}P T / H R \\
(s)\end{array}$ & $\begin{array}{l}\text { Heparin (ordered) } \\
\text { (units } / 24 \mathrm{~h})\end{array}$ & $\begin{array}{l}\text { Heparin (infused) } \\
\text { (units } / 24 h)\end{array}$ & $\begin{array}{l}\text { Coumarin } \\
(m g / 24 h)\end{array}$ & $\begin{array}{l}\text { Factors } \\
(\%)\end{array}$ \\
\hline $\begin{array}{l}0 \\
1 \\
2 \\
3 \\
4 \\
5\end{array}$ & $\begin{array}{l}51 \cdot 2 \\
63 \cdot 6 \\
73 \cdot 0 \\
48 \cdot 0 \\
50 \cdot 1 \\
60 \cdot 4\end{array}$ & $\begin{array}{l}29.0 \\
32.6 \\
37.5 \\
39.9 \\
40.9 \\
46.2\end{array}$ & $\begin{array}{r}- \\
16 \cdot 2 \\
21 \cdot 6 \\
10 \cdot 4 \\
5 \cdot 4 \\
23.9\end{array}$ & $\begin{array}{l}11.9 \\
21.6 \\
22.9 \\
19.4 \\
19.0 \\
19.5\end{array}$ & $\begin{array}{l}11.9 \\
18.7 \\
21.9 \\
20.6 \\
18.4 \\
19.2\end{array}$ & $\begin{array}{l}28800 \\
28800 \\
24000 \\
17900 \\
16000 \\
12200\end{array}$ & $\begin{array}{c}28600 \\
28600 \\
20240 \\
16300 \\
15940 \\
1060 \dagger\end{array}$ & $\begin{array}{r}10 \\
10 \\
5 \\
5 \\
5 \\
5\end{array}$ & $\begin{array}{l}\text { II } 63 \text {, VII } 3, \text { X } 37 \\
\text { II } 49 \text {, VII } 5, \text { X } 20 \\
\text { II } 27, \text { VII } 8, \text { X } 4\end{array}$ \\
\hline
\end{tabular}

Patient aPTT therapeutic range $=43 \cdot 5-72 \cdot 5 \mathrm{~s}$.

$\mathrm{hr}(\%)=\frac{\text { coumarin prolongation }}{\text { optimal prolongation }} \times 100=\frac{\text { coumarin prolongation }}{22 \cdot 2} \times 100$.

*Warfarin sodium.

†Discontinued.

aPTT = activated partial thromboplastin time.

$\mathrm{PT}=$ prothrombin time.

$/ \mathrm{HR}=$ after heparin removal . 
Table 2 Use of the heparin reduction algorithm in a patient with long coumarin induction interval: patient 4

\begin{tabular}{|c|c|c|c|c|c|c|c|c|}
\hline Day & $\underset{(s)}{a P T T}$ & $\begin{array}{l}a P T T / H R \\
(s)\end{array}$ & $\begin{array}{l}h r \\
(\%)\end{array}$ & $\begin{array}{l}P T \\
(s)\end{array}$ & $\begin{array}{l}P T / H R \\
(s)\end{array}$ & $\begin{array}{l}\text { Heparin (ordered) } \\
\text { (units } / 24 \text { h) }\end{array}$ & $\begin{array}{l}\text { Heparin (infused) } \\
\text { (units } / 24 h \text { ) }\end{array}$ & $\begin{array}{l}\text { Coumarin* } \\
(m g / 24 h)\end{array}$ \\
\hline $\begin{array}{l}0 \\
1 \\
2 \\
3 \\
4 \\
5 \\
6 \\
7 \\
8\end{array}$ & $\begin{array}{l}54 \cdot 6 \\
63 \cdot 7 \\
59 \cdot 8 \\
84 \cdot 4 \\
64 \cdot 2 \\
50 \cdot 2 \\
55 \cdot 6 \\
47 \cdot 9 \\
57 \cdot 4\end{array}$ & $\begin{array}{l}31 \cdot 7 \\
33 \cdot 5 \\
32 \cdot 9 \\
36 \cdot 2 \\
35 \cdot 8 \\
42 \cdot 3 \\
46 \cdot 9 \\
46 \cdot 4 \\
52 \cdot 8\end{array}$ & $\begin{array}{l}-7 \cdot 9 \\
\overline{11} \cdot 8 \\
\frac{26}{26} \cdot 7 \\
\frac{20 \cdot 1}{25} \cdot 8\end{array}$ & $\begin{array}{r}9.8 \\
10.2 \\
10.7 \\
12.7 \\
13.1 \\
14.6 \\
13.9 \\
15.7 \\
19.8\end{array}$ & $\begin{array}{r}9 \cdot 6 \\
9 \cdot 9 \\
10 \cdot 3 \\
11 \cdot 9 \\
12 \cdot 8 \\
14 \cdot 5 \\
14 \cdot 1 \\
15 \cdot 2 \\
19 \cdot 2\end{array}$ & $\begin{array}{ll}25 & 440 \\
25 & 440 \\
23 & 455 \ddagger \\
23 & 455 \\
20 & 690 \\
20 & 690 \ddagger \\
15 & 400 \\
12 & 480 \\
- & \end{array}$ & $\begin{array}{cc}25 & 200 \\
24 & 400 \\
24 & 100 \\
21 & 260 \\
20690 \\
17500 \\
12900 \\
10600 \\
6000 \dagger\end{array}$ & $\begin{array}{l}10 \\
10 \\
15 \\
15 \\
20 \\
15 \\
20 \\
17 \cdot 5 \\
10\end{array}$ \\
\hline
\end{tabular}

Patient aPTT therapeutic range $=47 \cdot 6-79 \cdot 3 \mathrm{~s}$.

$\mathrm{hr}(\%)=\frac{\text { coumarin prolongation }}{54 \cdot 6-31 \cdot 7} \times 100=\frac{\text { coumarin prolongation }}{22 \cdot 9} \times 100$.

*Warfarin sodium.

†Discontinued.

$\ddagger$ No change calculated from preceeding interval.

aPTT = activated partial thromboplastin time.

$\mathrm{PT}=$ prothrombin time.

$/$ HR $=$ after heparin removal.

exchanger was equilibrated in Owren's veronal buffer $(\mathrm{pH} 7 \cdot 35)$ and poured into disposable polypropylene columns with built-in filter discs (Quik Sep, California Scientific, San Jose, CA). The final packed column height was $1.0 \mathrm{~cm}$. Columns were

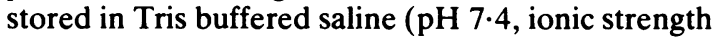
-0.12 at $4^{\circ} \mathrm{C}$ ) until used. The columns as used in the study removed a maximum of 25 units of heparin per millilitre of plasma without appreciably altering coagulation factor assays or global tests as described elsewhere. ${ }^{5}$

\section{Results}

The ideal execution of the algorithm in a hypothetical patient being maintained at a level of $1.9 x$ the baseline aPTT is shown in the Figure. In contrast, the relatively uncomplicated execution of the HRA in patient 3 is presented as the exemplary case. At the $\mathrm{n}=\mathrm{o}$ sampling interval the aPTT/HR indicated that heparin was responsible for $22.2 \mathrm{~s}$ of the patient's $51.2 \mathrm{~s}$ aPTT (Table 1). The pationt's therapeutic range was calculated before the administration of $10 \mathrm{mg}$ of warfarin sodium while the heparin infusion rate was maintained at 28600 units $/ 24 \mathrm{~h}$. The influence of the vitamin $\mathrm{K}$ antagonist was clearly manifest at time $n=1$, when the PT and PT/HR were already within the therapeutic range with a factor VII level of $3 \%$. By contrast, the aPTT/HR did not become therapeutic until day 5 , when all prothrombin group factors assayed were therapeutic.

Patient 4 showed an exceedingly long induction and stabilisation with oral anticoagulants, which was probably the result of the concurrent administration of broad spectrum antibiotics for pneumonia. On

Table 3 Application of the heparin reduction algorithm to the management of coumarins when heparin is overrange. Subject no 5

\begin{tabular}{|c|c|c|c|c|c|c|c|c|c|}
\hline Day & $\underset{(s)}{a P T T}$ & $\underset{(s)}{a P T T / H R}$ & $\begin{array}{l}h r \\
(\%)\end{array}$ & $\begin{array}{l}P T \\
(s)\end{array}$ & $\begin{array}{l}P T / H R \\
(s)\end{array}$ & $\begin{array}{l}\text { Heparin (ordered) } \\
\text { (units } / 24 \text { h) }\end{array}$ & $\begin{array}{l}\text { Heparin (infused) } \\
\text { (units } / 24 h)\end{array}$ & $\begin{array}{l}\text { Coumarin } \\
(m g / 24 h)\end{array}$ & $\begin{array}{l}\text { Factors } \\
(\%)\end{array}$ \\
\hline $\begin{array}{l}0 \\
1\end{array}$ & $\begin{array}{l}73 \cdot 3 \\
71 \cdot 7\end{array}$ & $\begin{array}{l}32 \cdot 8 \\
37 \cdot 4\end{array}$ & $\overline{11} \cdot 4$ & $\begin{array}{l}12 \cdot 0 \\
11 \cdot 2\end{array}$ & $\begin{array}{l}11 \cdot 2 \\
11 \cdot 2\end{array}$ & $\begin{array}{l}25200 \\
25200\end{array}$ & $\begin{array}{l}25200 \\
25200\end{array}$ & $\begin{array}{l}10 \\
10\end{array}$ & \multirow[t]{2}{*}{$\begin{array}{l}\text { II } 65, \text { VII } 25 \text {, } \\
\text { IX } 48, \text { X } 44\end{array}$} \\
\hline $\begin{array}{l}2 \\
3 \\
4 \\
5 \\
6\end{array}$ & $\begin{array}{l}50 \cdot 4 \\
98 \cdot 0 \\
98 \cdot 5 \\
87 \cdot 2 \\
64 \cdot 4\end{array}$ & $\begin{array}{l}30 \cdot 5 \\
35 \cdot 6 \\
46 \cdot 0 \\
45 \cdot 4 \\
54 \cdot 2\end{array}$ & $\begin{array}{l}-6.9 \\
25 \cdot 7 \\
-20.2\end{array}$ & $\begin{array}{l}14 \cdot 4 \\
21 \cdot 3 \\
22 \cdot 7 \\
23 \cdot 9 \\
25 \cdot 0\end{array}$ & $\begin{array}{l}13.7 \\
18.0 \\
20.8 \\
22.3 \\
24.8\end{array}$ & $\begin{array}{ll}22 & 320 \ddagger \\
22 & 320 \\
20 & 640 \\
15 & 336 \\
-\end{array}$ & $\begin{array}{l}24040 \\
23600 \\
23800 \\
19000 \\
t\end{array}$ & $\begin{array}{l}10 \\
7 \cdot 5 \\
7 \cdot 5 \\
8 \cdot 75 \\
8 \cdot 75\end{array}$ & \\
\hline
\end{tabular}

Subject aPTT therapeutic range $=48 \cdot 9-82 \cdot 0$.

$\mathrm{hr}(\%)=\frac{\text { coumarin prolongation }}{73 \cdot 3-32 \cdot 8} \times 100=\frac{\text { coumarin prolongation }}{40 \cdot 5} \times 100$.

*Warfarin sodium.

†Discontinued.

¥Calculated no change from preceeding interval.

aPTT = activated partial thromboplastin time

$\mathrm{PT}=$ prothrombin time.

$/ H R=$ after heparin removal. 
days 2 and 4 the HRA calculations indicated that heparin coverage should not be reduced because the aPTT/HR was actually shorter than the previous day (Table 2). It should be noted that the amount of heparin received by the patient as verified from input and output records was somewhat less than ordered, even though the trend was in the desired downward direction. The aPTT/HR and PT/HR did not become therapeutic in this patient until the eighth day of coumarin treatment.

The third individual (patient 5) was reported by the clinical management team to have an exquisite sensitivity to heparin. While the aPTT exceeded the therapeutic range on three of seven days during the changeover to coumarins, there was no evidence of haemorrhage or thrombosis (Table 3). The rate at which heparin was reduced by the hospital staff was actually less than was indicated by HRA calculations. In this instance the aPTT/HR became therapeutic on the sixth day of coumarin treatment, or three days after the PT/HR (PT).

\section{Discussion}

It has long been recognised that three phase treatment of venous thromboembolic disease with heparin and coumarins alone and in combination is highly effective. ${ }^{910}$ The traditional laboratory surveillance plans for heparin and coumarins administered individually are quite functional and furnish information that can be used to adjust drug dosages within tolerances that safeguard against the dangerous complications of recurrent thrombosis and haemorrhage. ${ }^{11}$

When these antithrombotic agents are administered simultaneously, however, the monitoring schemes fail to provide the requisite data necessary for the rational incremental withdrawal of heparin as the pharmacological effect of the coumarins becomes therapeutic. These shortcomings are due to the insensitivity of the PT to heparin and the fact that the intrinsic and extrinsic pathways are dissimilar with respect to the vitamin $\mathrm{K}$ dependent factors IX and VII, respectively. For this reason, the most difficult and most hazardous interval in the anticoagulant treatment of venous thromboembolic disease is managed by trial and error. The realisation that the aPTT/HR surveillance plan permitted the clinician for the first time to observe directly the developing coumarin effect prompted the formulation of the refinement known as the HRA.

Use of the HRA necessitates a familiarity with the fundamental tenets of the aPTT/HR plan..$^{5}$ Anion exchange heparin removal technology must be available for application on a routine basis. In addition, the institution has to identify aPTT reagents suitable for use with plasma from patients treated with anion exchange. ${ }^{12}$ Definition of the operational configuration of the HRA resulted from a careful evaluation of the ways in which anticoagulation is managed by the physician. A $24 \mathrm{~h}$ sampling interval was selected for this reason. Shorter cycles may be used with the plan should a more critical adjustment of heparin protection be required. Intervals longer than $24 \mathrm{~h}$ seem unreasonable owing to rapid development of the coumarin effect in most patients.

The success of the HRA as a therapeutic regimen in the management of patients 4 and 5 clearly shows that difficulties in the regulation of both the heparin and coumarin effect can be effectively dealt with so as to minimise the likelihood of complications. The new level of responsiveness provided by the HRA is a substantial improvement over the empirical transition plan as it permits heparin to be decreased, increased, or maintained in direct relation to the effect of the oral anticoagulant. While it was not stressed, the duration of the transition interval from heparin to coumarins should ideally increase with use of the HRA because the plan is based on the aPTT/HR. Such an increase is predictable because the $t 1 / 2$ disappearance time of factor IX $(17-40 \mathrm{~h})$ is considerably greater than that of factor VII (about $5 \mathrm{~h}$ ) in the one stage prothrombin time based surveillance schemes. It is readily seen that this is not a disadvantage in managing patients like patient 3 because the life span of factor IX more closely approximates that of factors $\mathrm{X}$ and II than factor VII. The potential problem of longer hospital stays can be readily circumvented by initiating oral anticoagulant treatment within one to four days of the stabilisation of heparin dosage as is now often done in many institutions.

In summary, the HRA integrates in a clinically meaningful way the aPTT/HR manoeuvre's capacity to provide information about the antithrombotic protection afforded by three anticoagulant effects - that is, (a) heparin, (b) coumarin, and (c) heparin + coumarin. By contrast, in the traditional plan there can be no informed reciprocal adjustment of the dosages of heparin and coumarin. The data provided by PT - that is, anion exchange prothrombin time or dilutional prothrombin time-even when coupled with that about heparin and coumarin from the aPTT cannot be correlated since the tests monitor separate pathways and have distinctive value scales. The use of the laboratory based HRA should make the management of the change from heparin to coumarin a more secure undertaking for all clinicians charged with the care of patients with venous thromboembolic disease. A determination of the effectiveness of the HRA in reducing the com- 
plications of haemorrhage and repeat thrombosis remains to be made.

\section{References}

' Israels E. Partial thromboplastin time in the presence of heparin. Am J Clin Pathol 1982;77:321-4.

${ }^{2}$ Owren PA. Thrombotest. A new method for controlling anticoagulant therapy. Lancet 1959;ii:754-8.

${ }^{3}$ Owren PA. Control of anticoagulant therapy. Arch Int Med 1963;III: $240-58$

${ }^{4}$ Branson HE, Slater LM, Anderson MG, Pirkle H. Prothrombin time after heparin removal: application to monitoring simultaneous anticoagulation with heparin and coumarins. Am J Clin Pathol 1979;71:665-7.

${ }^{5}$ Branson HE, Gupta N, Slater LM, Fagin A, Armentrout SA. The activated partial thromboplastin time after heparin removal (aPPT/HR) in a new scheme of anticoagulant monitoring. Am J Hematol 1982;12:411-8.

- Williams WJ. Thrombosis. In: Williams WJ, Beutler E, Erslev AJ, Lichtman MA, eds. Hematology. New York: McGraw-
Hill, 1983: 1480.

' Denson KWE. Appendixes 1 and 2. In: Biggs R, ed. Human blood coagulation, haemostasis and thrombosis. Oxford: Blackwell Scientific, 1976:655-750.

${ }^{8}$ Thompson AR, Counts RB. Removal of heparin and protamine from plasma. J Lab Clin Med 1976;88:922-9.

" Barritt DW, Jordan SC. Anticoagulant drugs in the treatment of pulmonary embolism: a controlled clinical trial. Lancet 1960;1:1309-12.

${ }^{10}$ Hull RB, Delmore T, Genton E, et al. Warfarin sodium versus low dose heparin in long-term treatment of venous thrombosis. N Engl J Med 1979;301:855-8.

"Kelton JG, Hirsh J. Bleeding associated with antithrombotic therapy. Sem Hematol 1980; 17:259-91.

${ }^{12}$ Branson HE, Sapra R, Roohk V, Fagin A, Arronte M. Use of the activated partial thromboplastin time to monitor coumarin anticoagulation: analysis of the behavior of a commercial reagent. J Natl Med Assn 1983;75:61-4.

Requests for reprints to: Dr Herman E Branson, 10906

Oakwood Street, Silver Spring, MD 20901, USA. 